Original Article

\title{
A STUDY ON THE EFFECT OF LOW MOLECULAR WEIGHT ON POTASSIUM HOMEOSTASIS IN PATIENTS ADMINISTERED HEPARIN FOR THROMBOPROPHYLAXIS IN A TERTIARY CARE HOSPITAL
}

\author{
S. SHANMUGAPRIYA*1, K. BHUVANESWARI ${ }^{2}$, K. RASHMI ${ }^{3}$ \\ 1,2Department of Pharmacology, ${ }^{3}$ Final year MBBS, PSG Institute of Medical Sciences and Research, Coimbatore 641004, Tamilnadu, India \\ Email: somasundaram999@rediffmail.com
}

Received: 28 Feb 2017 Revised and Accepted: 20 Apr 2017

\begin{abstract}
Objective: Low molecular weight heparin (LMWH) is an indirect thrombin inhibitor used clinically as an anticoagulant for thromboprophylaxis of patients at risk of deep vein thrombosis. The study was done to assess the magnitude of rise in serum potassium after administration of low molecular weight heparin comparing enoxaparin with dalteparin and to evaluate the frequency of clinically significant hyperkalemia in the
\end{abstract} population studied.

Methods: The study was done as a prospective non-randomized observational study in a population of 32 patients started on heparin for thromboprophylaxis for deep venous thrombosis, pulmonary thromboembolism or stroke.

Results: A statistically significant elevation in serum potassium was seen in patients treated with LMWH (p $=0.007$ ). The magnitude of rise in potassium was significantly higher in enoxaparin $(\mathrm{p}=0.008)$ than the dalteparin group $(\mathrm{p}=0.447)$. A clinically relevant hyperkalemia of $>5 \mathrm{mEq} / \mathrm{l}$ was seen in $25 \%$ of the population studied. Other important associations detected from the study were that the advancing age and increasing dose could be potential risk factors contributory to an accentuated rise in serum potassium which may culminate in clinically significant hyperkalemia.

Conclusion: The study has highlighted that the likelihood of rise in potassium levels during LMWH therapy necessitates monitoring of serum potassium.

Keywords: Serum potassium, Enoxaparin, Dalteparin, Hyperkalemia

(C) 2017 The Authors. Published by Innovare Academic Sciences Pvt Ltd. This is an open access article under the CC BY license (http://creativecommons.org/licenses/by/4.0/) DOI: http://dx.doi.org/10.22159/ijpps.2017v9i6.18191

\section{INTRODUCTION}

Heparin is a parenteral anticoagulant that acts as indirect thrombin inhibitor and is extensively used for treatment and prophylaxis of several thromboembolic conditions in clinical practice. Heparin is a heterogeneous mixture of sulfated mucopolysaccharides whose biologic activity is dependent upon the endogenous anticoagulant antithrombin. Antithrombin inhibits clotting factor proteases, especially thrombin (IIa), IXa, and Xa by forming equimolar stable complexes with them.

Hyperkalemia is an electrolyte abnormality associated with the use of heparin therapy in patients. It is a condition wherein the serum potassium is elevated above the normal range (Normal: 3.5-5 $\mathrm{mEq} / \mathrm{l})$. The incidence of heparin-induced hyperkalemia in hospitalized patients is estimated to be $1-10 \%$ [1]. The risk of heparin-induced hyperkalemia is increased many folds in patients with renal insufficiencies and in the elderly population [2]. Thus it is important for the physician to be aware of this complication and ensure appropriate monitoring of serum potassium levels during heparin therapy.

Unfractionated heparin (UFH) has a high molecular weight in the range of 5000-30,000 Daltons. In contrast, low molecular weight heparin (LMWH) is shorter chain fractions which inhibit activated factor X with a lesser effect on thrombin than the UFH. LMWH is as efficacious as UFH with more predictable therapeutic effect and lesser adverse effects. LMWH offers many advantages such as weight-based dosing, lower frequency of injections, eliminating the need for laboratory monitoring in addition to a decreased risk of heparininduced thrombocytopenia and osteoporosis. Therefore LMWH preparations are more commonly used in the current clinical scenario.

Heparin therapy can induce hypoaldosteronism by inhibiting the enzyme involved in the synthesis of angiotensin 11 receptors in the adrenal gland. This, in turn, produces natriuresis and hyperkalemia. Heparin can produce hyperkalemia within 4-7 d of beginning treatment [3]. The risk of hyperkalemia is increased in patients with chronic renal failure, diabetes mellitus, and elderly people [2]. While most physicians are aware of heparin-induced thrombocytopenia, heparin-induced hyperkalemia is less commonly recognised [4].

Though there are many case reports on unfractionated heparininduced hyperkalemia in literature, there are very few prospective studies evaluating the effect of LMWH on serum $\mathrm{K}^{+}$levels. Therefore our study was designed to evaluate the frequency of occurrence of a heparin-induced increase in serum $\mathrm{K}^{+}$levels in hospitalized patients receiving $\mathrm{LMWH}$ for thromboprophylaxis. We chose to compare the change in $\mathrm{K}^{+}$electrolyte levels due to enoxaparin with that of dalteparin as enoxaprin and dalteparin are the two commonly used low molecular weight heparins in patient care.

\section{MATERIALS AND METHODS}

This was a hospital based prospective open-label, non-randomized study. The institutional human ethics committee (IHEC) approved the protocol of the study. (Approval number: 13/361). The study was done in 32 patients started on low molecular weight heparin in our tertiary care teaching hospital. Patients who were admitted and started on LMWH, either enoxaparin or dalteparin were included for the study, based on inclusion and exclusion criteria.

All patients, aged $>30 \mathrm{y}$, both males and females with normal baseline potassium value and started on low molecular weight heparin, either enoxaparin or dalteparin for thromboprophylaxis in ICU or inpatient care were included for the study after obtaining a written informed consent. Patients with chronic renal insufficiencies or altered renal function tests, on pre-existing potassium supplementation or receiving drugs which can increase $\mathrm{K}^{+}$level like spironolactone, ACE inhibitors, angiotensin receptor blockers, NSAIDS including cyclooxygenase 2 inhibitors, trimethoprim, amiloride, triamterene, pentamidine, nafamostat, renin inhibitors, digoxin and related glycosides, mannitol, beta-blockers, succinylcholine and other drugs known to alter the potassium levels 
were excluded by a detailed evaluation of the list of concomitant drugs. Children, pregnant and lactating women were also excluded from the study. In addition, patients whose baseline potassium value was not estimated or missing were not included in the study.

After obtaining a written informed consent, data on demographic details, the diagnosis, the indication for heparin, other coadministered drugs and the serum electrolyte levels were obtained. The baseline $\mathrm{K}^{+}$level was collected from the case record of the patient. Another $\mathrm{K}^{+}$level estimation was done at the end of day 4 of starting heparin. Blood collection was done meticulously by a trained lab technician using standard venipuncture technique; taking care to avoid tourniquet, fist-clenching, inappropriate needle diameter, excessive force during aspiration and other maneuvers which could result in hemolysis or falsely elevated potassium values. Potassium estimation was done in the biochemistry laboratory by ion selective electrode method using potentiometry. Any documentation of symptoms pertaining to hyperkalemia during heparin therapy recorded in the case record was also noted. Data regarding the patients who developed clinically significant hyperkalemia requiring a change in therapy or other measures taken control the hyperkalemia was also collected. The data collected was entered into Excel and statistical analysis was done using SPSS software version 19.

Table 1: Baseline demographic data of the participants

\begin{tabular}{lll}
\hline S. No. & Parameter & Measured values \\
\hline 1 & Mean Age & 58.5 y \\
2 & Male female ratio & $14: 18$ \\
3 & Patients treated with LMWH & 32 \\
4 & Patients treated with Enoxaparin & $17(53 \%)$ \\
5 & Patients treated with Dalteparin & $15(47 \%)$ \\
\hline
\end{tabular}

Table 2: Paired t-test to compare the means of the serum potassium before and after administration of heparin across different age groups

\begin{tabular}{|c|c|c|c|c|c|c|c|}
\hline \multirow{3}{*}{$\begin{array}{l}\text { Groups } \\
\text { (n= no of patients) }\end{array}$} & \multicolumn{4}{|c|}{ Serum potassium level (mEq/l) } & \multicolumn{2}{|c|}{ Paired difference of before and after values } & \multirow[t]{3}{*}{ p-value } \\
\hline & \multicolumn{2}{|c|}{ Before } & \multicolumn{2}{|c|}{ After } & \multirow[t]{2}{*}{ Mean } & \multirow{2}{*}{ \pm Standard deviation } & \\
\hline & Mean & \pm SD & Mean & \pm SD & & & \\
\hline $31-60$ y $(n=13)$ & 3.87 & 0.436 & 4.20 & 0.794 & -0.329 & 0.526 & 0.065 \\
\hline $61-80$ y $(n=19)$ & 3.83 & 0.736 & 4.13 & 0.669 & -0.295 & 0.430 & 0.015 \\
\hline
\end{tabular}

$\mathrm{n}=32$; Data expressed as mean $\pm \mathrm{SD}$

\section{RESULTS}

A total number of 32 patients participated in this study. The mean age of the study participants was found to be $58.5 \pm 14.5$ y (table 1 ). About $59 \%$ of the patients were in the age group between $61-80 \mathrm{y}$ and the rest (41\%) were 31-60 y aged. The occurrence of serum potassium elevation, according to the study, was found to be significantly higher in the patients of 61 to 80 y age group, unlike the $31-60$ y age group subjects in whom the rise in potassium level after treatment was not statistically significant (table 2).

Amongst the study participants, $44 \%$ were males and 56\% were females (table 1). Among the patients started on low molecular weight heparin therapy, around 53\% received enoxaparin while $47 \%$ of patients received dalteparin (fig. 1). The mean potassium level done at the end of $4 \mathrm{~d}$ after starting treatment with low molecular weight heparin, showed a demonstrable increase compared to baseline and a paired t-test revealed a statistically significant difference $(\mathrm{p}=0.007)$ in the serum potassium before and after treatment with heparin (fig. 2).

Considering the change in serum potassium levels in the enoxaparintreated group and the group treated with dalteparin, it was observed that both groups had a definite increase in the mean potassium levels after commencing treatment. However, it was found using paired ttest that a statistically significant increase in the serum potassium level was demonstrable only for the enoxaparin group $(p=0.008)$, but not for the dalteparin group $(\mathrm{p}=0.447)$ (table 3 ).

In addition, a correlation between increases in serum potassium with that of the dose administered could be identified for both groups as shown in the graphical representations fig. 3,4 .

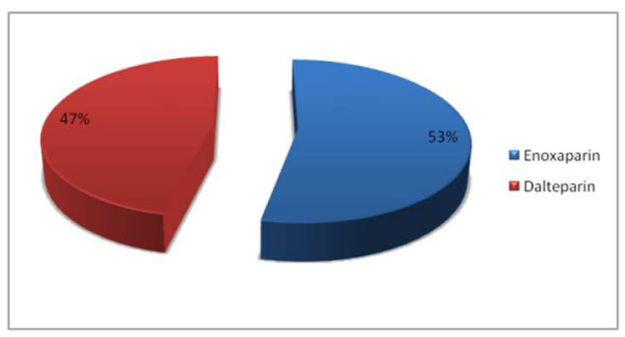

Fig. 1: Frequency distribution of the patients started on low molecular weight (enoxaparin, dalteparin)

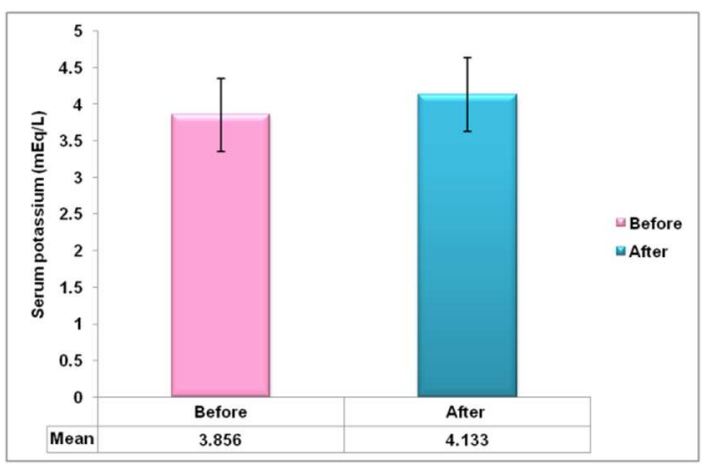

Fig. 2: Serum Potassium expressed as mean \pm SD before and after treatment with low molecular weight heparin

Table 3: Paired t-Test to show the statistically significant difference in the serum potassium values before and after treatment

\begin{tabular}{|c|c|c|c|c|c|c|c|}
\hline \multirow{3}{*}{$\begin{array}{l}\text { Groups } \\
\text { (n= no of patients) }\end{array}$} & \multicolumn{4}{|c|}{ Serum potassium level (mEq/l) } & \multicolumn{2}{|c|}{ Paired difference of before and after values } & \multirow[t]{3}{*}{ p-value } \\
\hline & \multicolumn{2}{|l|}{ Before } & \multicolumn{2}{|l|}{ After } & \multirow[t]{2}{*}{ Mean } & \multirow[t]{2}{*}{ \pm Standard deviation } & \\
\hline & Mean & \pm SD & Mean & \pm SD & & & \\
\hline LMWH (n=32) & 3.89 & 0.667 & 4.12 & 0.707 & -0.232 & 0.391 & 0.007 \\
\hline Enoxaparin $(\mathrm{n}=17)$ & 3.81 & 0.556 & 4.10 & 0.602 & -0.292 & 0.396 & 0.008 \\
\hline Dalteparin $(n=15)$ & 4.06 & 0.31 & 4.16 & 0.332 & -0.106 & 0.373 & 0.447 \\
\hline
\end{tabular}

Data expressed as mean \pm SD 


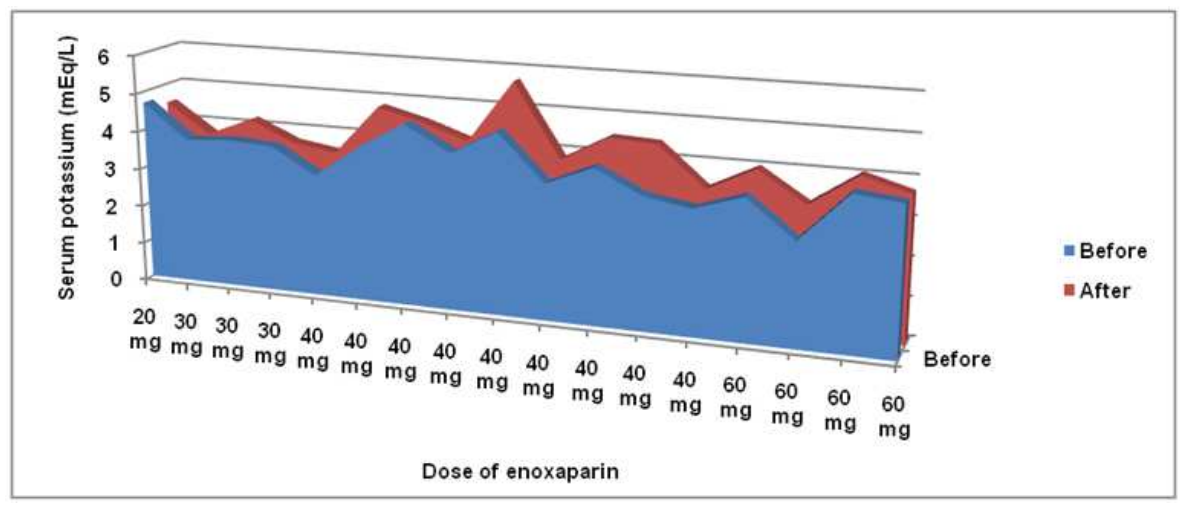

Fig. 3: Chart representing the rise in serum potassium levels across various doses of enoxaparin



Fig. 4: Chart representing the rise in serum potassium levels across various doses of dalteparin

Clinically relevant hyperkalemia (more than $5 \mathrm{mEq} / \mathrm{l}$ ) was noted in $8(25 \%)$ patients out of the 32 participants. Among these patients, $5(16 \%)$ were treated with dalteparin and $3(9 \%)$ were in the group treated with enoxaparin (fig. 5). Though around
$1 / 4^{\text {th }}$ of the patients in the study manifested with clinically relevant hyperkalemia, none of them was symptomatic or needed any specific measures to correct the electrolyte abnormality.

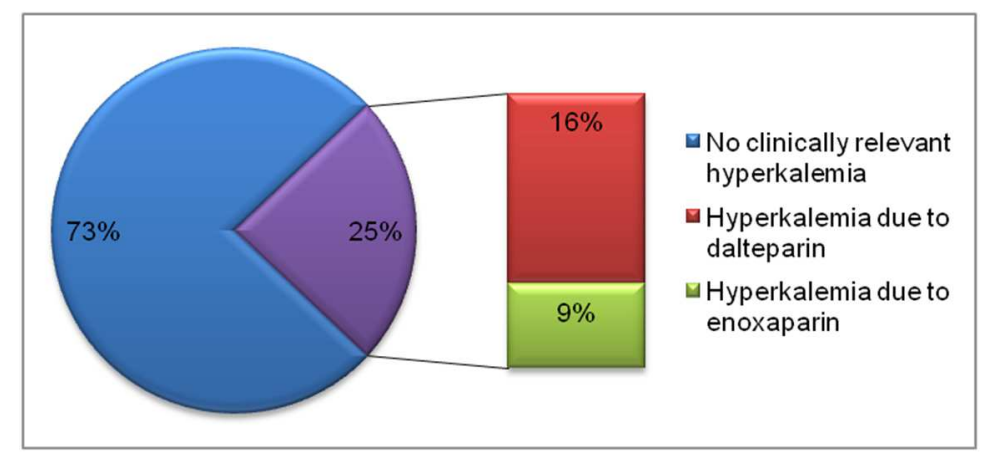

Fig. 5: Chart representing the frequency of patients who developed clinically significant hyperkalemia (>5 mEq/l)

\section{DISCUSSION}

In our study done in 32 patients started on low molecular weight heparin, there were $46 \%$ aged $31-60$ y whereas a larger proportion of patients, ie, $59 \%$ were in the age group of $61-80 \mathrm{y}$. This emphasises that there is an increased risk of thromboembolic disease as the age advances. This has been demonstrated in earlier studies. $[5,6]$ This is probably attributed to the increase in the prevalence of the risk factors for thromboembolic diseases with ageing.
In addition, it was established through the study results that increasing age is also associated with an increased risk of hyperkalemia with heparin therapy. This is evident from the statistically significant increase in serum potassium in the $61-80 \mathrm{y}$ age group ( $p=0.065)$, but not in the age group of 31-60 y ( $p=0.015)$ (table 3). The male-female ratio was 14: 18 indicating that a higher number of female patients had received heparin therapy during the study duration. Noteworthy as most of the female patients who participated in the study were in the postmenopausal age group but none of them was on any hormonal treatment. 
Using paired t-test, a statistically significant rise in serum potassium was demonstrated in the study population treated with LMWH ( $\mathrm{p}=$ 0.007). In the current clinical scenario, UFH has been largely replaced by the LMWH. This is quite understandable, particularly since there are plenty of advantages for LMWH over UFH like equal efficacy, increased bioavailability, and less frequent dosing. Also, monitoring of activated partial thromboplastin time is not required. It has been well brought out in this study that the low molecular weight heparin is also implicated in increasing the serum potassium levels like UFH.

The primary mechanism of heparin-induced hyperkalemia has been well established and it is due to suppression of aldosterone synthesis. This is mediated by a reversible effect on aldosterone through blockade of an enzymatic step in the synthesis and also the angiotensin II receptors in the adrenal gland. The most important mechanism of heparin-induced hypoaldosteronism involves a reduction in both number and affinity of angiotensin II receptors in the zona glomerulosa. Prolonged administration of heparin causes a marked reduction in the width of adrenal zona glomerulosa thereby leading to hyperkalemia and natriuresis. This reversible suppression of aldosterone occurs within a few days of initiation of therapy and is independent of either the anticoagulant effect or the route of administration. It has been reported that reduced aldosterone levels may be evident as early as $4^{\text {th }}$ day of initiation of heparin therapy [3]. Thus LMWH has effects similar to that of UFH on aldosterone $[7,8]$ and hence LMWH is also associated with increasing the risk of hyperkalemia in treated patients. This has also been brought out by our study result which is well in concordance with previous studies reported in the literature $[9,10]$.

It was found that among the LMWH, enoxaparin (53\%) was more commonly used than dalteparin for thromboprophylaxis $(47 \%)$. The definitive reason for this cannot be offered, especially because an extended literature search has shown multiple studies and metanalyses comparing UFH with LMWH, but head to head comparisons of the different LMW heparins are few in number and these have claimed similar compliance, health status, deep venous thrombosis, and bleeding rates with both [11] while some studies have brought out that switching patients from enoxaparin to dalteparin was cost-effective [12]. However the DETECT trial has established the hypothesis that dalteparin 5,000 units subcutaneous (SC) daily may not be clinically non-inferior to enoxaparin $30 \mathrm{mg} \mathrm{SC}$ twice daily for venous thromboembolism prophylaxis in high-risk population and until an adequately powered, prospective noninferiority trial is performed, enoxaparin is supported by level 1 evidence and should be the prophylactic agent of choice [13].

In our study, a statistically significant increase in the potassium level was seen only in the group treated with enoxaparin $(p=0.008)$ but not the dalteparin group $(p=0.447)$. Though the study infers a statistically higher risk of serum potassium elevation with enoxaparin in comparison to dalteparin, this cannot be translated to a definite clinical significance with certainty owing to two reasons. First is the lack of sufficient sample size and secondly, on account of the remarkable finding that a higher proportion of patients in dalteparin group (15\%) had clinically relevant hyperkalemia ( $>5$ $\mathrm{mEq} / \mathrm{l}$ ) compared to $9 \%$ in the enoxaparin group. An explanation for more number of patients manifesting hyperkalemia in the dalteparin group is probably due to the higher mean baseline value $(4.06 \pm 0.31)$ compared to that of enoxaparin group $(3.81 \pm 0.55)$. This is in accordance to a study which has shown that baseline potassium level reflects pretreatment aldosterone level and hence its value has a predictive role for assessing the susceptibility to develop hyperkalemia [10].

Interestingly, the results of the study also revealed an association of a larger rise in potassium levels being associated with higher doses of both the low molecular heparins studied. This is substantiated by the fact that the higher doses of enoxaparin 40 and $60 \mathrm{mg}$ produced a greater elevation of serum potassium levels as compared to the lower doses of 20,30 mg and this observation was replicated in the groups on dalteparin $2500 \mathrm{U}$ versus $5000 \mathrm{U}$ as well (fig. 3,4 ). Thus the adverse effect is found to be dose dependent and the possible explanation of this observation stems from an accentuated aldosterone suppression by higher doses of heparin leading to an augmented rise in serum potassium levels.

Thus the study has clearly delineated that significant elevation of serum $\mathrm{K}+$ is seen in patients treated with low molecular weight heparin and that the magnitude of the rise in potassium to clinically relevant hyperkalemia $(>5 \mathrm{mEq} / \mathrm{l})$ occurs in approximately onequarter of the patients studied. This signifies the need for adequate monitoring of the serum K+while on LMWH for thromboprophylaxis.

The limitation of the study is the small sample size. Therefore it is recommended that the study results have to be confirmed in larger trials in order to enable extrapolation of the results to the larger patient population treated with heparin clinically.

\section{CONCLUSION}

The study has highlighted the magnitude of changes in potassium levels in patients receiving low molecular weight heparin for thromboprophylaxis. In addition to the known risk factors like renal failure and concurrently administered drugs, the study has made it evident that advancing age and increasing dose could potentially contribute to the development of hyperkalemia with low molecular weight heparin therapy. Thus the study results help the clinicians to potentially predict the patients likely to experience this electrolyte disturbance; stratify their risk and manage them appropriately.

\section{ACKNOWLEDGEMENT}

We thank the Indian Council of Medical Research (ICMR) for funding the research project under STS scheme.

\section{CONFLICT OF INTERESTS}

There are no conflicts of interest

\section{AUTHORS CONTRIBUTION}

Author 1 was involved in concept, design, data collection, analysis and interpretation and author 2 were involved in concept, design and data interpretation. The third author was involved in the design, data collection and analysis of the research project.

\section{CONFLICT OF INTERESTS}

\section{Declared none}

\section{REFERENCES}

1. Kuijvenhoven MA, Haak EA, Gombert-Handoko KB, Crul M. Evaluation of the concurrent use of potassium-influencing drugs as risk factors for the development of hyperkalemia. Int J Clin Pharm 2013;35:1099-104.

2. Bacon NC. Heparin-induced disturbance of potassium homeostasis. Q J Med 1997;90:725.

3. Thomas CM, Thomas J, Smeeton F, Leatherdale BA. Heparininduced hyperkalemia. Diabetes Res Clin Pract 2008;80:e7-8.

4. Torres OH, Hernandez N, Francia E, Barcelo M, Mateo J, Ruiz D. Effect of prophylactic treatment with low-molecular-weight heparin bemiparin sodium on serum potassium levels: a prospective observational study. Drugs Aging 2010;27:399-406.

5. Olson NC, Cushman M, Lutsey PL, McClure LA, Judd S, Tracy $\mathrm{RP}$, et al. Inflammation markers and incident venous thromboembolism: the REasons for geographic AND racial differences in the stroke (REGARDS) cohort. J Thromb Haemost 2014;12:1993-2001.

6. Aronow WS, Schoenfeld MR, Gutstein H. Frequency of thromboembolic stroke in persons greater than or equal to $60 \mathrm{y}$ of age with extracranial carotid arterial disease and/or mitral annular calcium. Am J Cardiol 1992;70:123-4.

7. Gheno G, Savarino C, Vellar S, Cinetto L. Heparin-induced lifethreatening hyperkalemia. Ann Ital Med Int 2002;17:51-3.

8. Gonzalez-Martin G, Díaz-Molinas MS, Martínez AM, Ortiz M. Heparin-induced hyperkalemia: a prospective study. Int J Clin Pharmacol Ther Toxicol 1991;29:446-50.

9. Gheno G, Cinetto L, Savarino C, Vellar S, Carraro M, Randon M. Variations of serum potassium level and risk of hyperkalemia in inpatients receiving low-molecular-weight heparin. Eur J Clin Pharmacol 2003;59:373-7. 
10. Bengalorkar GM, Sarala N, Venkatrathnamma PN, Kumar TN. Effect of heparin and low-molecular-weight heparin on serum potassium and sodium levels. J Pharmacol Pharmacother 2011;2:266-9.

11. Chiou-Tan FY, Garza H, Chan KT, Parsons KC, Donovan WH, Robertson CS, et al. Comparison of dalteparin and enoxaparin for deep venous thrombosis prophylaxis in patients with spinal cord injury. Am J Phys Med Rehabil 2003;82:678-85.

12. Krotenberg R, Adler U, Pomeranz B, Miller JD, Russell MW. Dalteparin vs. enoxaparin as prophylaxis for deep-vein thrombosis after total hip or knee arthroplasty: a retrospective analysis. Am J Phys Med Rehabil 2001;80:889-95.
13. Slavik RS, Chan E, Gorman SK, de Lemos J, Chittock D, Simons $\mathrm{RK}$, et al. Dalteparin versus enoxaparin for venous thromboembolism prophylaxis in acute spinal cord injury and major orthopedic trauma patients: 'DETECT' trial. J Trauma 2007;62:1075-81.

\section{How to cite this article}

- S Shanmugapriya, K Bhuvaneswari, K Rashmi. A study on the effect of low molecular weight on potassium homeostasis in patients administered heparin for thromboprophylaxis in a tertiary care hospital. Int J Pharm Pharm Sci 2017;9(6):85-89. 